\title{
Bovine lactoferricin B induces apoptosis of human gastric cancer cell line AGS by inhibition of autophagy at a late stage
}

\author{
W.-R. Pan, ${ }^{* 1}$ P.-W. Chen, $\dagger^{1}$ Y.-L. S. Chen, ${ }^{*}$ H.-C. Hsu, ${ }^{*}$ C.-C. Lin, ${ }^{*}$ and W.-J. Chen ${ }^{* 2}$ \\ *Department of Biotechnology and Animal Science, National llan University, 1 Shen-Lung Road Sec.1, Ilan 26047, Taiwan, Republic of China \\ †Department of Nursing, St. Mary's Junior College of Medicine, Nursing and Management, 100, Lane 265, Sang-Sing Road Sec. 2 , \\ Sang-Sing 26644, Ilan County, Taiwan, Republic of China
}

\section{ABSTRACT}

Gastric cancer is one of the most common malignant cancers, with poor prognosis and high mortality rates worldwide. Therefore, development of an effective therapeutic method without side effects is an urgent need. It has been reported that cationic antimicrobial peptides can selectively bind to negatively charged prokaryotic and cancer cell membranes and exert cytotoxicity without causing severe drug resistance. In the current study, we prepared a series of peptide fragments derived from bovine lactoferrin and evaluated their anticancer potency toward the gastric cancer cell line AGS. Cell viability assay revealed that a 25-AA peptide fragment, lactoferricin B25 (LFcinB25), exhibited the most potent anticancer capability against AGS cells. Lactoferricin B25 selectively inhibited AGS cell growth in a dose-dependent manner, exhibiting a half-maximal inhibitory concentration $\left(\mathrm{IC}_{50}\right)$ value of $64 \mu M$. Flow cytometry showed a notable increment of the sub- $\mathrm{G}_{1}$ populations of the cell cycle, indicating the induction of apoptosis by LFcinB25. Western blot analysis further revealed that upon LFcinB25 treatment for 2 to $6 \mathrm{~h}$, apoptosis-related caspases-3, 7, 8, 9, and poly(ADP-ribose) polymerase (PARP) were cleaved and activated, whereas autophagy-related LC3-II and beclin-1 were concomitantly increased. Thus, both apoptosis and autophagy are involved in the early stage of LFcinB25-induced cell death of AGS cells. However, upon treatment with LFcinB25 for 12 to $24 \mathrm{~h}$, LC3-II began to decrease, whereas cleaved beclin-1 increased in a time-dependent manner, suggesting that consecutive activation of caspases cleaved beclin-1 to inhibit autophagy, thus enhancing apoptosis at the final stage. These findings provide support for future application of LFcinB25 as a potential therapeutic agent for gastric cancer.

Received July 24, 2013.

Accepted September 2, 2013.

${ }^{1}$ These two authors contributed equally to this paper.

${ }^{2}$ Corresponding author: wjchen@niu.edu.tw
Key words: bovine lactoferricin B, gastric cancer, apoptosis, autophagy

\section{INTRODUCTION}

Gastric cancer is one of the most common causes of cancer-related mortality worldwide, especially in Asian countries (Kelley and Duggan, 2003; Guggenheim and Shah, 2013). In general, the main curative therapies for gastric cancer are surgery and chemotherapy, which are generally only successful if the cancer is diagnosed at an early stage (Baeza et al., 2001; Longley et al., 2003; Cascinu et al., 2004). The 5-yr survival of gastric cancer patients is currently estimated at approximately $30 \%$; therefore, novel treatment strategies to improve prognosis are urgently needed.

Lactoferrin ( $\mathbf{L F})$ is an $80-\mathrm{kDa}$ iron-binding glycoprotein mainly present in milk and other exocrine secretions (Baveye et al., 1999). Many physiological roles have been ascribed to LF, including antimicrobial and immunomodulatory activities (Levay and Viljoen, 1995; Chierici, 2001; Farnaud and Evans, 2003; Legrand and Mazurier, 2010). Lactoferrin is also considered to play important roles in the regulation of cell growth and differentiation (Kanyshkova et al., 2001). Treatment of the protein with pepsin is reported to yield a hydrolysate which, although unable to bind iron, remains antibacterially active (Tomita et al., 1991). An active peptide, lactoferricin B (LFcinB), was subsequently isolated from the hydrolysate and identified as residues 17 to 41 of bovine LF (bLF; Bellamy et al., 1992b). Lactoferricin B is a 25-AA cationic antimicrobial peptide (AMP) with an amphipathic, antiparallel $\beta$-sheet structure (Hwang et al., 1998). It has a single disulfide bond and no iron-binding capacity, and is active against a broad spectrum of microorganisms, including bacteria (Bellamy et al., 1993), fungi (Cascinu et al., 2004), and viruses (Andersen et al., 2001). Previous studies have shown that LFcinB also possesses potent in vitro and in vivo anticancer activity (Yoo et al., 1997a,b). Like other cationic AMP with anticancer activity, LFcinB is believed to interact with negatively charged regions on 
cancer cells and subsequently disturb cancer cell membranes via the insertion of hydrophobic AA residues into the lipid bilayer (Hoskin and Ramamoorthy, 2008). Recently, researchers have shown that LFcinB selectively induces apoptosis in a range of human leukemia and carcinoma cell lines without harming normal mammalian cells (Mader et al., 2005, 2007; Furlong et al., 2006). The same group showed that LFcinB kills human neuroblastoma cells by a membranolytic mechanism and inhibits the growth of neuroblastoma xenografts in nude mice (Eliassen et al., 2006). They also showed that LFcinB induces caspase-independent apoptosis in Raji and Ramos human B-lymphoma cells and extends the survival of nude mice bearing B-lymphoma xenografts (Furlong et al., 2010). Lactoferricin B used in this study was designated as LFcinB25, compared with 2 other truncated fragments, LFcinB11 and LFcinB6 (Table 1). These 2 peptides both contain the antimicrobial core sequence RRWQWR (Tomita et al., 1994) and can adopt a well-defined amphipathic structure when bound to SDS micelles (Schibli et al., 1999).

In the current study, we initially assessed the cytotoxicity of bovine lactoferrin acid hydrolysate (bLFh) and 3 peptide fragments derived from LFcinB against the gastric cancer cell line AGS. Lactoferricin B25, showing the most potent anticancer capability, was then investigated further for its anticancer mechanisms. To the best of our knowledge, this is the first report describing the interplay between apoptosis and autophagy in LFcinB-induced cell death of cancer cells. These findings provided support that LFcinB25 may have chemotherapeutic potential for the treatment of gastric cancer.

\section{MATERIALS AND METHODS}

\section{Preparation and Characterization of bLF, bLFh, and LFcinB}

Bovine LF was purchased from Glanbia Nutritionals (Monroe, WI), and bLFh was prepared by acid-pepsin hydrolysis as described previously (Chen et al., 2004,
2013). Briefly, bLF was dissolved in distilled water at $5 \%$ (wt/vol) and the $\mathrm{pH}$ was adjusted to 2 to 3 . Porcine pepsin was added to prepare a final concentration of $\mathrm{bLF}$ of $3 \%$ (wt/wt), and digestion was conducted at $37^{\circ} \mathrm{C}$ for $4 \mathrm{~h}$. The reaction was terminated by heating the solution at $80^{\circ} \mathrm{C}$ for $15 \mathrm{~min}$, followed by neutralization to $\mathrm{pH} 7.0$ by the addition of $1 \mathrm{~N} \mathrm{NaOH}$. The remaining insoluble peptides were removed by centrifugation at $15,000 \times g$, and the supernatant was immediately used in experiments or stored at $-20^{\circ} \mathrm{C}$ until use. The LFcinB peptides are fragments derived from pepsin hydrolysis of bLF and have been identified as cationic AMP (Bellamy et al., 1992a). In this study, 3 LFcinB (LFcinB25, LFcinB11, LFcinB6) were synthesized on a solid-phase peptide synthesizer (model 433A; Applied Biosystems, Foster City, CA) as previously described (Chou et al., 2008) using the FastMoc chemistry [2-(1H-benzotriazol-1-yl)-1,1,3,3-tetramethyluronium (HBTU)/1-hydroxybenzotriazole (HOBt) activation strategy]. The crude peptides were cleaved from the resin by $95 \%$ trifluoroacetic acid, separated by filtration, precipitated from diethyl ether, and lyophilized to yield white solid powders. Peptides were analyzed and purified by reversed-phase (RP)-HPLC, and were further characterized by matrix-assisted laser desorption/ionization time-of-flight (MALDI-TOF) mass spectrometry, as previously described (Chou et al., 2008). The purity of bLF, bLFh, and LFcinB was analyzed by $12.5 \%$ SDS-PAGE using the Protean III System (Bio-Rad, Hercules, CA), as previously described (Laemmli, 1970).

\section{Cell Lines and Cell Culture}

The human gastric cancer cell line AGS was kindly provided by Y. L. Chen (National Ilan University, Taiwan). The mouse fibroblast cell line 3T3 (BCRC 60071) was obtained from Bioresources Collection and Research Center (Hsin Chu, Taiwan). The 3T3 cells were maintained in Dulbecco's modified Eagle medium containing $10 \%$ fetal bovine serum, and the AGS cells were maintained in RPMI-1640 medium containing $10 \%$

Table 1. Characteristics of lactoferricin B peptide fragments used in the current study

\begin{tabular}{|c|c|c|c|c|}
\hline \multirow[b]{2}{*}{ Peptide $^{1}$} & \multirow[b]{2}{*}{ Amino acid sequence ${ }^{2}$} & \multirow[b]{2}{*}{ Charge } & \multicolumn{2}{|c|}{ Molecular weight } \\
\hline & & & Calculated & Observed \\
\hline LFcinB6 & RRWQWR & +3 & 987.13 & 987.14 \\
\hline LFcinB11 & RRWQWRMKKLG & +5 & $1,544.89$ & $1,544.90$ \\
\hline LFcinB25 & FKCRRWQWRMKKLGAPSITCVRRAF & +8 & $3,125.82$ & $3,125.84$ \\
\hline
\end{tabular}


fetal bovine serum at $37^{\circ} \mathrm{C}$ in a humidified atmosphere containing $5 \% \mathrm{CO}_{2}$, as previously described (Chen et al., 2012).

\section{Cell Viability Assay}

The viability of the cells after treatment with various AMP was evaluated using 3-(4,5-dimethylthiazol2-yl)-2,5-diphenyltetrazolium bromide (MTT) assays performed in triplicate. Briefly, cells were plated at a density of 2,500 cells/well in 96-well plates, permitted to adhere for 12 to $18 \mathrm{~h}$, and then washed with PBS. Solutions were always prepared freshly by dissolving $1 \times$ PBS or drugs in culture medium. After $24 \mathrm{~h}$ of exposure, the peptide-containing medium was removed, the cells were washed with PBS, and fresh medium was added. The cells in each well were then incubated in culture medium with $500 \mu \mathrm{g} / \mathrm{mL}$ MTT for $2 \mathrm{~h}$. After the medium was removed, $200 \mu \mathrm{L}$ of dimethyl sulfoxide (DMSO) was added to each well. Absorbance at $570 \mathrm{~nm}$ of the maximum was detected by a multimode microplate reader SpectraMax M2 (Molecular Devices, Sunnyvale, CA). The absorbance for DMSO-treated cells was considered to be $100 \%$. The results were determined by 3 independent experiments.

\section{Flow Cytometric Analysis of Cell Cycle}

After treatment with LFcinB25, AGS cells were harvested and washed twice with PBS and fixed in $70 \%$ cold ethanol at $4^{\circ} \mathrm{C}$ overnight. Before analysis, cells were washed twice with PBS containing $1 \%$ BSA, resuspended with $400 \mu \mathrm{L}$ of PBS, and treated with 100 $\mu \mathrm{g} / \mathrm{mL}$ RNase A (Roche Diagnostics, Indianapolis, IN) and $20 \mu \mathrm{g} / \mathrm{mL}$ propidium iodide (PI; Sigma Corp., St. Louis, MO). After incubation for $30 \mathrm{~min}$ at $37^{\circ} \mathrm{C}$, the cells were subjected to DNA content analysis. The PI fluorescence was analyzed by using a FACScalibur flow cytometer (Becton Dickinson, Franklin Lakes, NJ). Data from at least 10,000 cells were analyzed with FlowJo software (Becton Dickinson). Cell cycle distributions were calculated with FlowJo software.

\section{Western Blot Analysis}

Approximately $1 \times 10^{6}$ AGS cells were cultured in $60-\mathrm{mm}^{2}$ dishes and then incubated in $64 \mu \mathrm{M}$ LFcinB25 for the indicated time. The cells were lysed on ice with $200 \mu \mathrm{L}$ of protein extraction buffer $(50 \mathrm{~m} M$ Tris-HCl, $\mathrm{pH}$ 7.5, $0.5 \mathrm{M} \mathrm{NaCl}, 5 \mathrm{mM} \mathrm{MgCl}, 0.5 \%$ Nonidet P-40, 1 $\mathrm{m} M$ phenylmethylsulfonyl fluoride, $1 \mu \mathrm{g} / \mathrm{mL}$ pepstatin, and $50 \mu \mathrm{g} / \mathrm{mL}$ leupeptin) and centrifuged at 12,000 $\times g$ at $4^{\circ} \mathrm{C}$ for $10 \mathrm{~min}$. The protein concentration of the cell lysates was measured with a protein assay (Bio-Rad) following the manufacturer's instructions. Aliquots (20 $\mu \mathrm{g})$ of the cell lysates were separated by 12.5 to $15 \%$ SDSPAGE (Bio-Rad). Resolved proteins were then transferred to polyvinylidene fluoride (PVDF) membranes. Filters were blocked with $5 \%$ nonfat milk overnight and 1:500 to 1:1,000 dilutions of primary antibodies for $1 \mathrm{~h}$ at room temperature. Membranes were washed with 3 times with $0.05 \%$ Tween-20 and incubated with 1:5,000 dilution of horseradish peroxidase-conjugated secondary antibody for $1 \mathrm{~h}$ at room temperature, and then visualized with an enhanced chemiluminescence plus chemiluminescence system (Millipore, Billerica, MA).

\section{RESULTS}

\section{SDS-PAGE Analysis of $b L F, b L F h$, and LFcinB}

Bovine LF, bLFh, and LFcinBs were analyzed by SDS-PAGE (Figure 1). The purity of bLF ( $>80-\mathrm{kDa}$ band) was around $75 \%$, whereas bLFh showed major protein bands of 3 to $5 \mathrm{kDa}$. The 3 synthetic peptides LFcinB25, LFcinB11, and LFcinB6 were prepared by solid-phase synthesis and purified by RP-HPLC to at least $95 \%$ homogeneity (Figure 1).

\section{Cytotoxicity of bLFh and LFcinB Against AGS Cell Line}

The AGS cell line was treated with different concentrations of bLFh and LFcinB for $24 \mathrm{~h}$, and then cell viability [reported as half-maximal inhibitory concentration $\left(\mathbf{I C}_{50}\right)$ values; Table 2] was determined using the MTT assay (Figure 2). Only cells treated with LFcinB25 exhibited reduced viability in a dose- and time-dependent manner, showing an $\mathrm{IC}_{50}$ value of 64 $\mu M$. The AGS cell line was less sensitive to LFcinB11, LFcinB6, and bLFh $\left(\mathrm{IC}_{50}\right.$ values $\left.>500 \mu M\right)$. The nontransformed murine fibroblast 3T3 line was less susceptible to all LFcinB peptides and bLFh tested, having significantly higher $\mathrm{IC}_{50}$ values $(>500 \mu M)$ compared with the AGS cell line. In summary, the peptide LF-

Table 2. Effect of lactoferricin B peptides (LFcinB6, B11, B25) and bovine lactoferrin hydrolysate (bLFh) on cell viability in gastric cancer cell line AGS and mouse fibroblast cell line 3T3

\begin{tabular}{lcc}
\hline & \multicolumn{2}{c}{$\mathrm{IC}_{50}(\mu M)^{1}$} \\
\cline { 2 - 3 } Peptide & AGS & $3 \mathrm{~T} 3$ \\
\hline LFcinB6 & $>500$ & $>500$ \\
LFcinB11 & $6500 \pm 4$ & $>500$ \\
LFcinB25 & $>500$ & $>500$ \\
bLFh & $>500$ \\
${ }^{1}$ IC $_{50}$ (half-maximal inhibitory concentration) values were assessed by \\
logarithmic extrapolation.
\end{tabular}




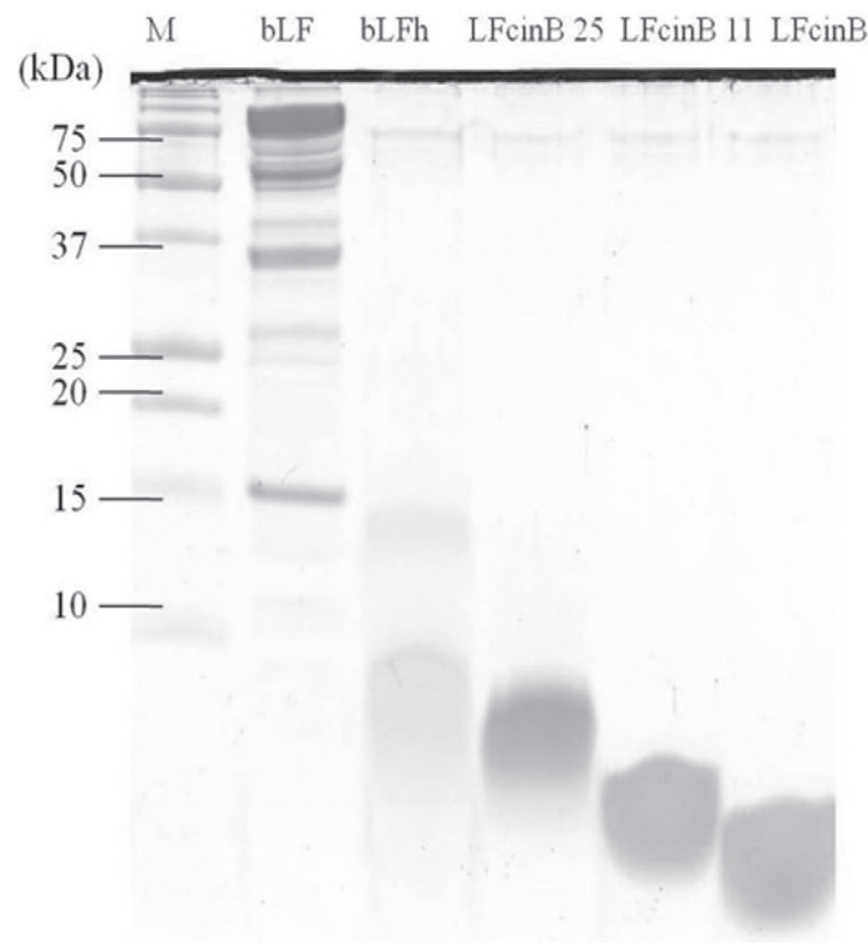

Figure 1. The purity of bovine lactoferrin (bLF), bLF acid hydrolysate (bLFh), and 3 lactoferricin B (LFcinB) peptides were analyzed by $15 \%$ Tricine SDS-PAGE and stained with Coomassie Brilliant Blue G-250. $\mathrm{M}=$ molecular weight marker.

cinB25 had a selective inhibitory effect on the viability of the AGS cell line compared with the 3T3 cell line, and was selected for further analysis of the anticancer mechanism.

\section{LFcinB25 Alters AGS Cell Morphology}

The AGS cells were treated with $64 \mu M$ LFcinB25 and cell morphology was assessed at $0,1,2,3,6,12$, and $24 \mathrm{~h}$ (Figure 3). After incubation for $1 \mathrm{~h}, \mathrm{LF}$ cinB25 was found at the cell membrane, and it induced the appearance of membrane-bound particles that were irregularly shaped and smaller than the untreated cells in a time-dependent manner. After the AGS cells had been incubated with the peptide for $24 \mathrm{~h}$, they had significantly shrunk in size and lost their capacity to adhere to the substratum.

\section{LFcinB25 Increases the Length of the Sub-G Phase as Revealed by Flow Cytometry}

To investigate whether LFcinB25 induced cytotoxicity of AGS cell line through the apoptotic pathway, flow cytometry was applied to monitor AGS cells in the absence or presence of $64 \mu M$ LFcinB25 for 0 to $24 \mathrm{~h}$, with visualization by PI staining. An increased

\section{(A) AGS}

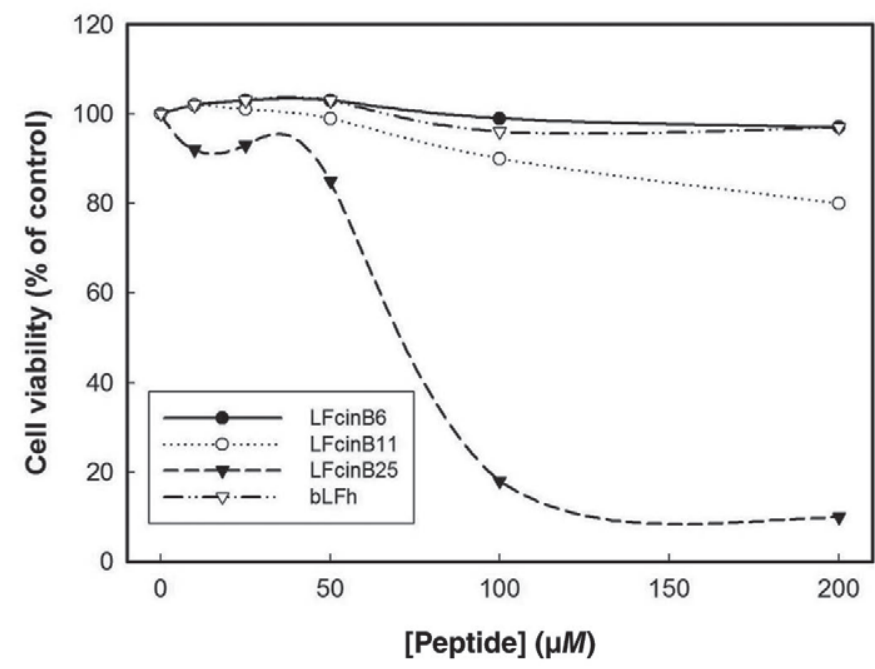

(B) $3 \mathrm{~T} 3$

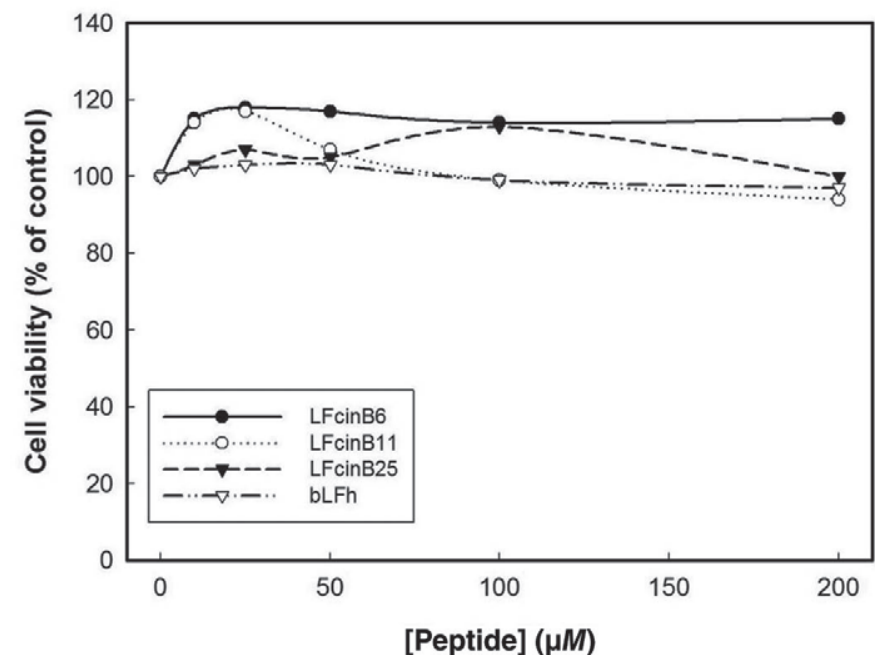

Figure 2. Effects of bovine lactoferrin acid hydrolysate (bLFh) and lactoferricin B (LFcinB) peptides on the cell viability of gastric cancer cell line AGS (A) and a nontransformed murine fibroblast cell line 3T3 (B). Cells were treated with different concentrations $(0$ to $200 \mu M)$ of bLFh and LFcinB peptides each for $24 \mathrm{~h}$, followed by an 3-(4,5-dimethylthiazol-2-yl)-2,5-diphenyltetrazolium bromide (MTT) assay. Each concentration was repeated in 8 wells for 3 independent experiments.

percentage of cells in the sub- $\mathrm{G}_{1}$ phase was observed in a time-dependent manner (Figure 4A, B), which is indicative of apoptotic cells.

\section{LFcinB25 Induced Both Apoptosis and Autophagy of AGS Cells in the Early Stage}

To further investigate whether LFcinB25 induced apoptosis of AGS cells through the caspase-dependent 

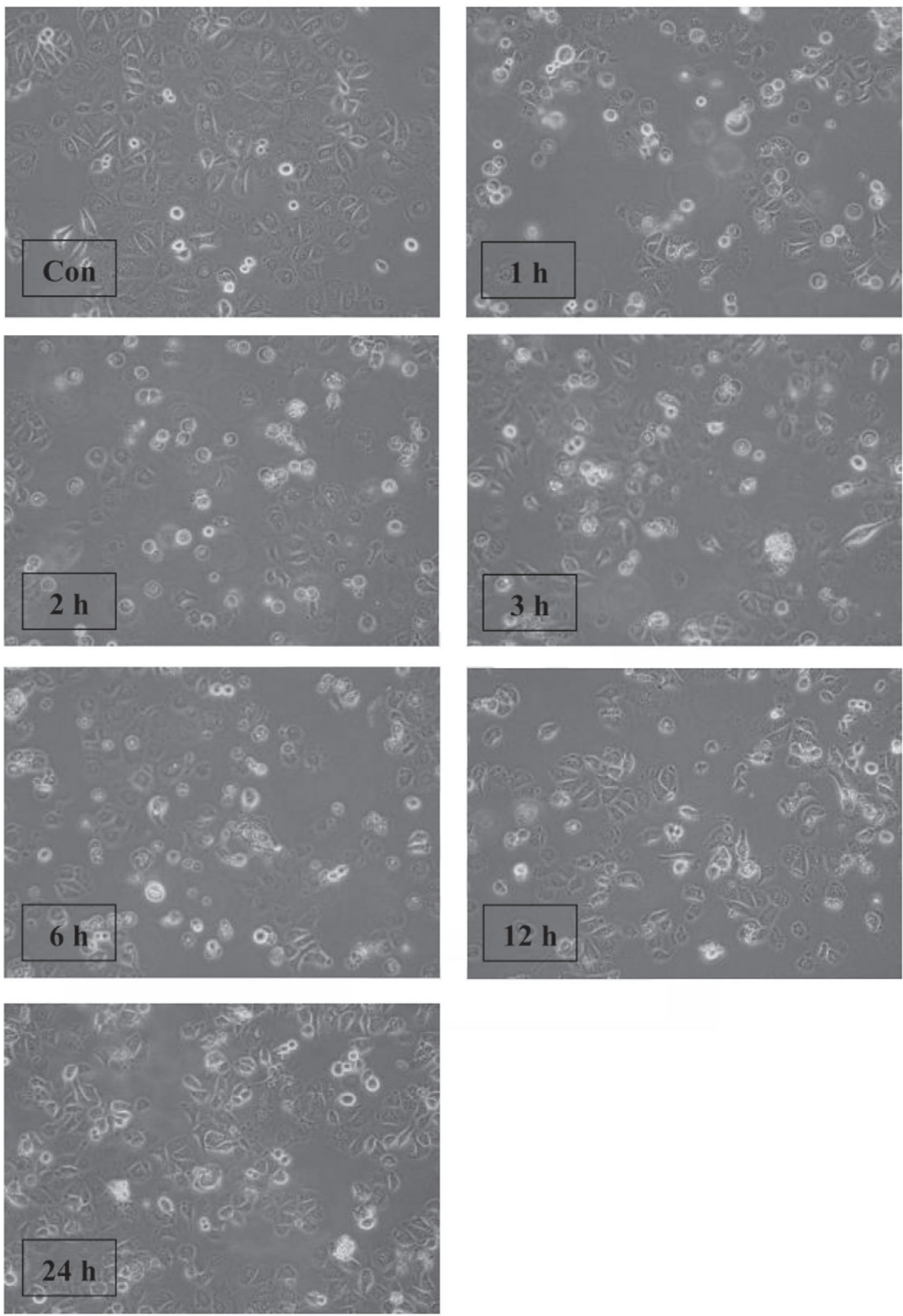

Figure 3. Effects of lactoferricin B25 (LFcinB25) on AGS cell (gastric cancer cell line) morphology. The AGS cells were treated with $64 \mu M$ LFcinB25, and cell morphology changes were assessed from 0 to $24 \mathrm{~h}$ using an inverted microscope with $200 \times$ magnification. 


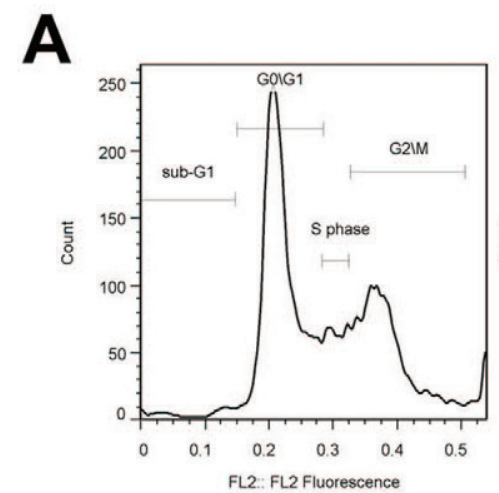

Control

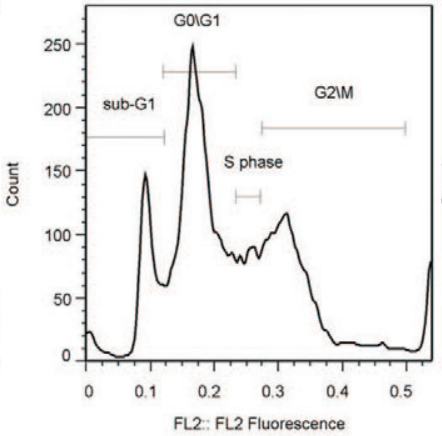

3H

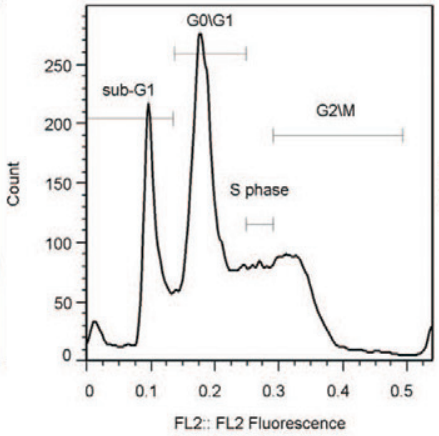

$6 \mathrm{H}$

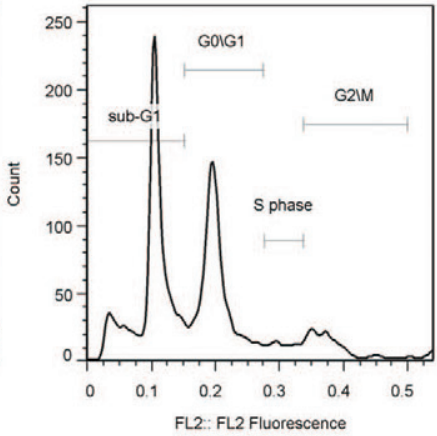

24H

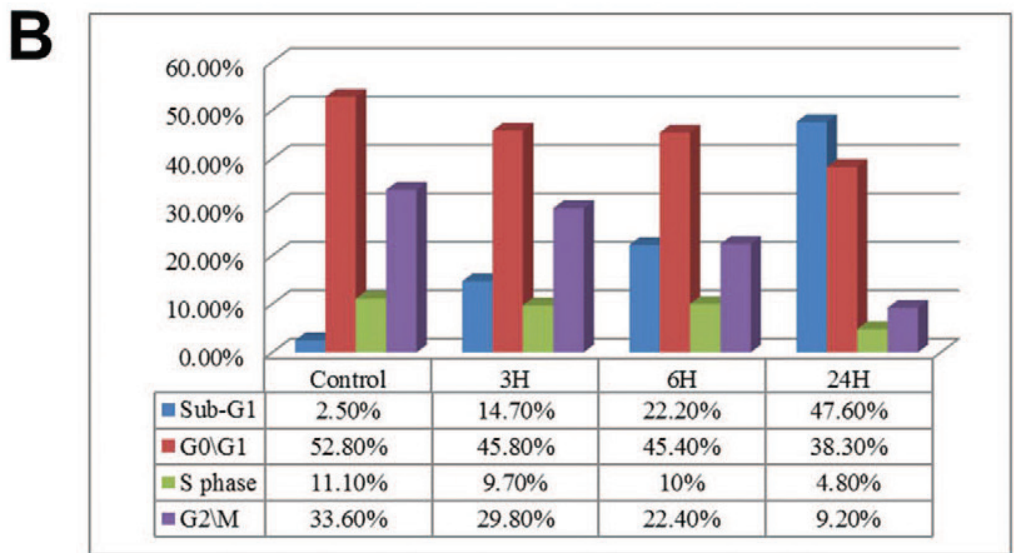

Figure 4. Lactoferricin B25 (LFcinB25) treatment of AGS cells (gastric cancer cell line) led to the accumulation of cells in the sub-G phase $_{1}$ (A and B) of the cell cycle. Flow cytometry was applied to analyze the AGS cell cycle in the absence or presence of LFcinB25 (64 $\mu M$ ) for 0 to $24 \mathrm{~h}$, with visualization by propidium iodide (PI) staining. Color version available in the online PDF.

pathway, we determined if caspases $3,7,8$, and 9 , and poly(ADP-ribose) polymerase (PARP) were cleaved in cells treated with the peptide. According to the results of Western blot analysis, these enzymes were all cleaved in a time-dependent manner (Figure 5), indicating that LFcinB25 induced caspase-dependent apoptosis of AGS cells via both intrinsic and extrinsic pathways. Interestingly, the autophagy-related proteins LC3-II and beclin-1 were found to concomitantly increase upon LFcinB25 treatment for 2 to $6 \mathrm{~h}$ (Figure 6). These results indicated that both apoptosis and autophagy were involved in the early stage of LFcinB25-induced cell death of AGS cells.

\section{LFcinB25-Induced Apoptosis of AGS Cells Was Enhanced by Inhibition of Autophagy at the Final Stage}

By examining the Western blot results, we found that upon LFcinB25 treatment of AGS cells for 12 to 24 h, LC3-II began to decrease, whereas cleaved beclin-1 increased in a time-dependent manner (Figure 6), sug- gesting that consecutive activation of caspases cleaved beclin-1 to inhibit autophagy, thus enhancing apoptosis of AGS cells at the final stage.

\section{DISCUSSION}

In the current study, the cationic AMP LFcinB25 had a potent effect on the antiproliferation of AGS cells, whereas no significant growth inhibitory effect was detected on 3T3 cells (Figure 2, Table 2). Generally, AMP exhibit selectivity for cancer and microbial cells primarily because of their elevated levels of negative membrane surface charge compared with noncancerous eukaryotic cells (Dennison et al., 2006). These cationic and amphipathic AMP are able to discriminate between neoplastic and non-neoplastic cells interacting specifically with negatively charged membrane components such as phosphatidylserine, sialic acid, or heparan sulfate, which differ between cancer and noncancer cells (Riedl et al., 2011). The anticancer activity of AMP normally occurs at micromolar levels but is not accompanied by significant levels of hemolysis or toxicity to other mam- 


\section{$\begin{array}{lllllllll}\text { Con } & 1 & 2 & 3 & 6 & 12 & 24 & 48 & (\mathrm{~h})\end{array}$}

Cytochrome C

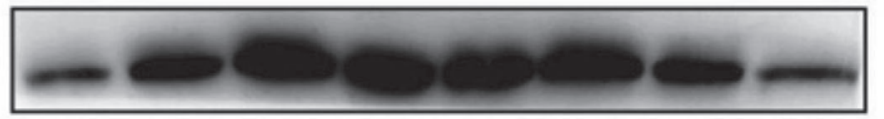

Cleaved Caspase 9

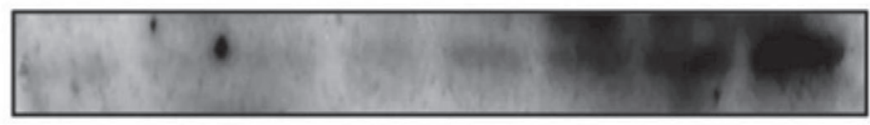

Pro Caspase 8
Cleaved Caspase 8

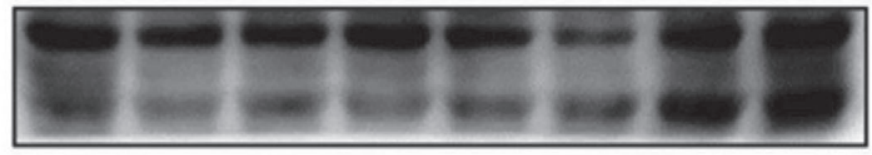

Pro Caspase 3

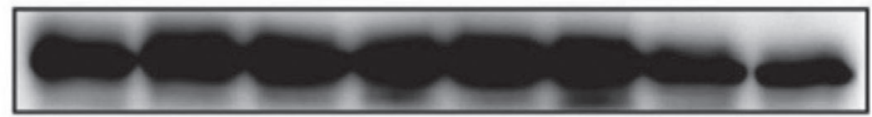

Cleaved Caspase3

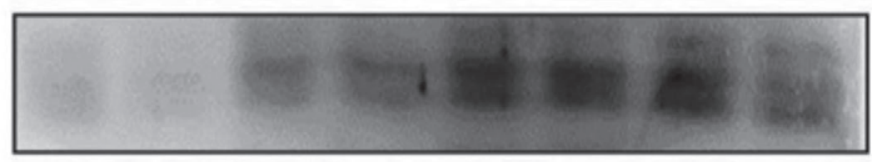

PARP

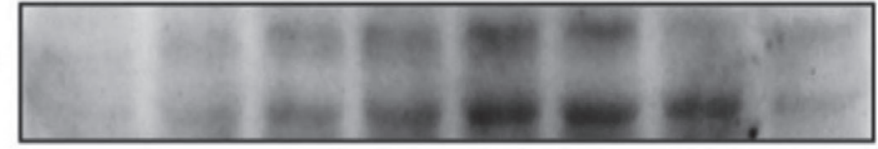

Pro Caspase 7

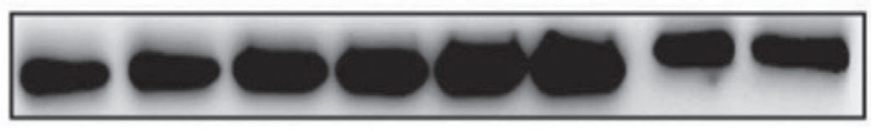

Cleaved Caspase 7

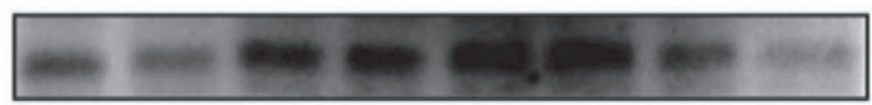

$\beta$-actin

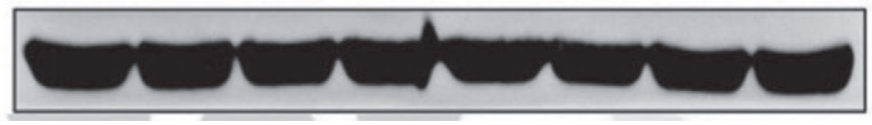

Figure 5. Western blot analysis for the activation of apoptosis-related proteins. The AGS cells (gastric cancer cell line) were treated with lactoferricin B25 $(64 \mu M)$ for 0 to $48 \mathrm{~h}$, and the cellular extracts were detected by Western blotting for cytochrome C, the pro- and cleaved forms of caspase-3, caspase-7, caspase-8, caspase-9, and poly(ADP-ribose) polymerase (PARP) to see if LFcinB25 induced apoptosis of AGS cells through the caspase-dependent intrinsic or extrinsic pathways. Lane Con = untreated control. $\beta$-Actin was used as internal control.

malian cells. In most cases, the mechanisms underlying such killing involve disruption of mitochondrial membrane integrity or that of the plasma membrane of the target tumor cells (Dennison et al., 2006). We thereby reasoned that differences in plasma membrane contents and mitochondrial membrane potential between the AGS and 3T3 cell lines may account for the differences in sensitivity to AMP. The peptides LFcinB11 and LFcinB6, although containing the antimicrobial core sequence RRWQWR, failed to kill AGS gastric cancer cells. Previous studies have shown that neither T-leukemia nor breast cancer cells were killed by free LFcinB6; however, fusogenic liposome-mediated delivery of LFcinB6 into the cytosolic compartment resulted in extensive DNA fragmentation that was dependent on cathepsin B and caspase activation (Richardson et al., 2009).
Previous studies have shown that LFcinB selectively induces apoptosis in a range of human leukemia and carcinoma cell lines via the reactive oxygen species-dependent loss of mitochondrial transmembrane potential and the sequential activation of caspase-2, caspase-9, and caspase-3, without harming normal mammalian cells (Mader et al., 2005; Furlong et al., 2006). In contrast, LFcinB kills human neuroblastoma cells by a membranolytic mechanism and inhibits the growth of neuroblastoma xenografts in nude mice (Eliassen et al., 2006). Furlong et al. (2010) also showed that LFcinB induced caspase-independent apoptosis in Raji and Ramos human B-lymphoma cells and extended the survival of immune-deficient mice bearing B-lymphoma xenografts. In the current study, Western blot analysis revealed that upon LFcinB25 treatment for 2 to $6 \mathrm{~h}$, apoptosis-related caspases 3, 7, 8, 9, and PARP were 


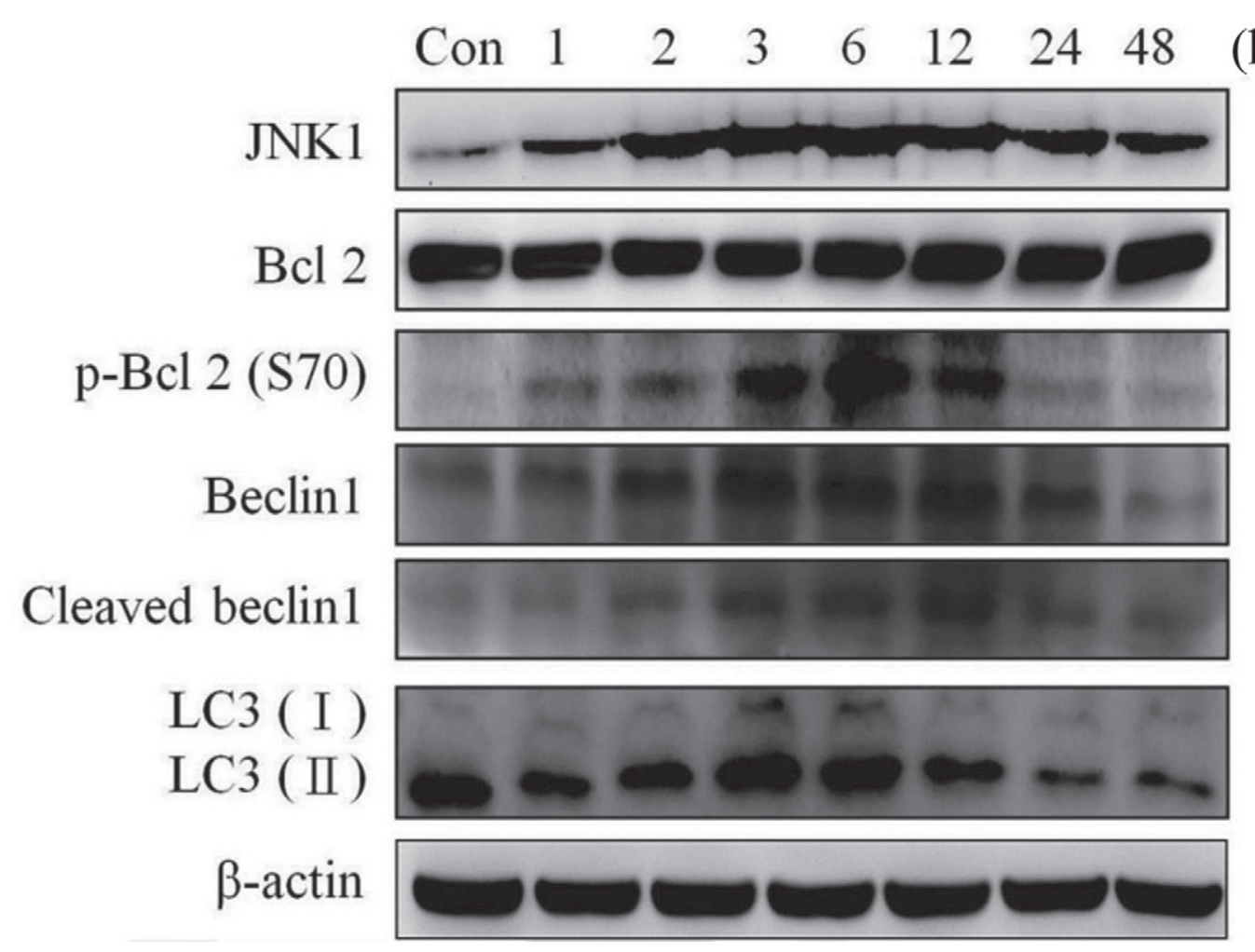

Figure 6. Western blot analysis for the activation of autophagy-related proteins. The AGS cells were treated with lactoferricin B25 (LFcinB25; $64 \mu M$ ) for 0 to $48 \mathrm{~h}$, and their cellular extracts were detected by Western blotting for JNK-1, Bcl-2, phosphorylated Bcl-2, Beclin 1, cleaved Beclin 1, LC3 I, and LC3 II to see if LFcinB25 induced autophagy of AGS cells. Lane Con = untreated control. $\beta$-Actin was used as internal control.

cleaved and activated (Figure 5), whereas autophagyrelated LC3-II and beclin-1 were concomitantly increased (Figure 6). These results indicated that both apoptosis and autophagy were involved in the early stage of LFcinB25-induced cell death of AGS cells. However, after treatment for 12 to $24 \mathrm{~h}$, LC3-II began to decrease and cleaved beclin-1 increased in a timedependent manner, suggesting that consecutive activation of caspases cleaved beclin-1 to inhibit autophagy, thus enhancing apoptosis of AGS cells at the final stage (Figure 6). To the best of our knowledge, this is the first report describing interplay between apoptosis and autophagy in LFcinB-induced cell death of cancer cells.

Autophagy is a catabolic process that is generally used by the cell as a mechanism for quality control and survival under stress conditions (Denton et al., 2012). Apoptosis and necrosis are the 2 major modes of cell death, the molecular mechanisms of which have been extensively studied. Importantly, autophagy has been linked to both types of cell death, serving either a prosurvival or prodeath function (Nikoletopoulou et al., 2013). A growing number of studies show that apoptosis induction is often associated with increased autophagy, indicating the existence of interplay between these 2 important cellular events (Fimia and Piacentini, 2010). It is clear that autophagy and apoptosis are strictly interconnected, as highlighted by the finding that the 2 pathways share key molecular regulators. Autophagy is an evolutionarily conserved stress response mechanism that often occurs in apoptosis-defective cancer cells and can protect against cell death ( $\mathrm{Li}$ et al., 2011). Beclin 1 plays a central role in autophagy by interacting with several cofactors to regulate the lipid kinase Vps-34 protein and promote formation of Beclin 1-Vps34-Vps15 core complexes, thereby inducing autophagy (Kang et al., 2011). Recent studies have suggested possible molecular mechanisms for crosstalk between autophagy and apoptosis. The well-characterized apoptosis guards Bcl-2 and Bcl-xL appear to be important factors in autophagy, inhibiting Beclin 1-mediated autophagy by binding to Beclin 1 (Giansanti et al., 2011). This interaction can be disrupted by JNK1-mediated phosphorylation of $\mathrm{Bcl}-2$, which results in the dissociation and activation of Beclin 1, thus facilitating autophagy (Shimizu et al., 2004, 2010; Zhou et al., 2011). In the current study, upon LFcinB25 treatment for 2 to $12 \mathrm{~h}$, concomitant increases of JNK-1, phosphorylated Bcl-2, and cleaved Beclin 1 could be observed by Western blot 
analysis (Figure 6), confirming the crosstalk between Bcl-2 and Beclin 1 and their roles in apoptosis and autophagy.

Previous studies have also shown that during chemotherapy-induced apoptosis of cancer cells, subsequent to cytochrome $\mathrm{C}$ release, autophagy is inhibited by caspase 8-mediated cleavage of Beclin 1 (Yu et al., 2004; Li et al., 2011). In the current study, upon LFcinB25 treatment for 6 to $12 \mathrm{~h}$ and following cytochrome $\mathrm{C}$ release (Figure 5), cleaved Beclin 1 began to increase (Figure 6 ), whereas caspase- 8 was activated during 12 to $24 \mathrm{~h}$ (Figure 5). Whether other caspases (i.e., caspase-3 or caspase-9) are responsible for Beclin 1 cleavage requires further investigation. Interestingly, caspase-mediated cleavage of Beclin 1 promotes crosstalk between apoptosis and autophagy.

\section{CONCLUSIONS}

The cationic AMP LFcinB25 exerts highly selective cytotoxicity against the AGS gastric cancer cell line via enhanced caspase-dependent apoptosis by inhibition of autophagy at the final stage. According to our results, Beclin 1 may be a useful target for inhibiting autophagy to sensitize LFcinB therapy for gastric cancer. Optimization of LFcinB using various strategies to enhance further selectivity is expected to yield novel anticancer drugs with chemotherapeutic potential for the treatment of gastric cancer.

\section{ACKNOWLEDGMENTS}

This work was supported in part by grants from the National Science Council (Taiwan, Republic of China): NSC 100-2311-B-197-001 to W.-J. Chen and NSC 1002313-B-562-001 to P.-W. Chen.

\section{REFERENCES}

Andersen, J. H., S. A. Osbakk, L. H. Vorland, T. Traavik, and T. J. Guttenberg. 2001. Lactoferrin and cyclic lactoferricin inhibit the entry of human cytomegalovirus into human fibroblasts. Antiviral Res. 51:141-149.

Baeza, M. R., T. O. Giannini, S. R. Rivera, P. Gonzalez, J. Gonzalez, E. Vergara, C. del Castillo, J. Madrid, and E. Vines. 2001. Adjuvant radiochemotherapy in the treatment of completely resected, locally advanced gastric cancer. Int. J. Radiat. Oncol. Biol. Phys. 50:645-650.

Baveye, S., E. Elass, J. Mazurier, G. Spik, and D. Legrand. 1999. Lactoferrin: A multifunctional glycoprotein involved in the modulation of the inflammatory process. Clin. Chem. Lab. Med. 37:281-286.

Bellamy, W., M. Takase, H. Wakabayashi, K. Kawase, and M. Tomita. 1992a. Antibacterial spectrum of lactoferricin B, a potent bactericidal peptide derived from the N-terminal region of bovine lactoferrin. J. Appl. Bacteriol. 73:472-479.

Bellamy, W., M. Takase, K. Yamauchi, H. Wakabayashi, K. Kawase, and M. Tomita. 1992b. Identification of the bactericidal domain of lactoferrin. Biochim. Biophys. Acta 1121:130-136.
Bellamy, W., H. Wakabayashi, M. Takase, K. Kawase, S. Shimamura, and M. Tomita. 1993. Killing of Candida albicans by lactoferricin $\mathrm{B}$, a potent antimicrobial peptide derived from the N-terminal region of bovine lactoferrin. Med. Microbiol. Immunol. (Berl.) 182:97-105.

Cascinu, S., M. Scartozzi, R. Labianca, V. Catalano, R. R. Silva, S. Barni, A. Zaniboni, A. D'Angelo, S. Salvagni, and G. Martignoni. 2004. High curative resection rate with weekly cisplatin, 5-fluorouracil, epidoxorubicin, 6S-leucovorin, glutathione, and filgastrim in patients with locally advanced, unresectable gastric cancer: A report from the Italian Group for the Study of Digestive Tract Cancer (GISCAD). Br. J. Cancer 90:1521-1525.

Chen, P. W., S. P. Ho, C. L. Shyu, and F. C. Mao. 2004. Effects of bovine lactoferrin hydrolysate on the in vitro antimicrobial susceptibility of Escherichia coli strains isolated from baby pigs. Am. J. Vet. Res. 65:131-137.

Chen, P. W., T. T. Jheng, C. L. Shyu, and F. C. Mao. 2013. Antimicrobial potential for the combination of bovine lactoferrin or its hydrolysate with lactoferrin-resistant probiotics against foodborne pathogens. J. Dairy Sci. 96:1438-1446.

Chen, Y. L., J. H. Li, C. Y. Yu, C. J. Lin, P. H. Chiu, P. W. Chen, C. C. Lin, and W. J. Chen. 2012. Novel cationic antimicrobial peptide GW-H1 induced caspase-dependent apoptosis of hepatocellular carcinoma cell lines. Peptides 36:257-265.

Chierici, R. 2001. Antimicrobial actions of lactoferrin. Adv. Nutr. Res. 10:247-269.

Chou, H. T., T. Y. Kuo, J. C. Chiang, M. J. Pei, W. T. Yang, H. C. Yu, S. B. Lin, and W. J. Chen. 2008. Design and synthesis of cationic antimicrobial peptides with improved activity and selectivity against Vibrio spp. Int. J. Antimicrob. Agents 32:130-138.

Dennison, S. R., M. Whittaker, F. Harris, and D. A. Phoenix. 2006. Anticancer $\alpha$-helical peptides and structure/function relationships underpinning their interactions with tumour cell membranes. Curr. Protein Pept. Sci. 7:487-499.

Denton, D., S. Nicolson, and S. Kumar. 2012. Cell death by autophagy: Facts and apparent artefacts. Cell Death Differ. 19:87-95.

Eliassen, L. T., G. Berge, A. Leknessund, M. Wikman, I. Lindin, C. Løkke, F. Ponthan, J. I. Johnsen, B. Sveinbjørnsson, P. Kogner, T. Flaegstad, and Ø. Rekdal. 2006. The antimicrobial peptide, lactoferricin B, is cytotoxic to neuroblastoma cells in vitro and inhibits xenograft growth in vivo. Int. J. Cancer 119:493-500.

Farnaud, S., and R. W. Evans. 2003. Lactoferrin-A multifunctional protein with antimicrobial properties. Mol. Immunol. 40:395-405.

Fimia, G. M., and M. Piacentini. 2010. Regulation of autophagy in mammals and its interplay with apoptosis. Cell. Mol. Life Sci. 67:1581-1588.

Furlong, S. J., J. S. Mader, and D. W. Hoskin. 2006. Lactoferricininduced apoptosis in estrogen-nonresponsive MDA-MB-435 breast cancer cells is enhanced by C6 ceramide and tamoxifen. Oncol. Rep. 15:1385-1390.

Furlong, S. J., J. S. Mader, and D. W. Hoskin. 2010. Bovine lactoferricin induces caspase-independent apoptosis in human B-lymphoma cells and extends the survival of immune-deficient mice bearing B-lymphoma xenografts. Exp. Mol. Pathol. 88:371-375.

Giansanti, V., A. Torriglia, and A. I. Scovassi. 2011. Conversation between apoptosis and autophagy: "Is it your turn or mine? Apoptosis 16:321-333.

Guggenheim, D. E., and M. A. Shah. 2013. Gastric cancer epidemiology and risk factors. J. Surg. Oncol. 107:230-236.

Hoskin, D. W., and A. Ramamoorthy. 2008. Studies on anticancer activities of antimicrobial peptides. Biochim. Biophys. Acta 1778:357-375.

Hwang, P. M., N. Zhou, X. Shan, C. H. Arrowsmith, and H. J. Vogel. 1998. Three-dimensional solution structure of lactoferricin B, an antimicrobial peptide derived from bovine lactoferrin. Biochemistry 37:4288-4298.

Kang, R., H. J. Zeh, M. T. Lotze, and D. Tang. 2011. The Beclin 1 network regulates autophagy and apoptosis. Cell Death Differ. 18:571-580.

Kanyshkova, T. G., V. N. Buneva, and G. A. Nevinsky. 2001. Lactoferrin and its biological functions. Biochemistry (Mosc.) 66:1-7. 
Kelley, J. R., and J. M. Duggan. 2003. Gastric cancer epidemiology and risk factors. J. Clin. Epidemiol. 56:1-9.

Laemmli, U. K. 1970. Cleavage of structural proteins during the assembly of the head of bacteriophage T4. Nature 227:680-685.

Legrand, D., and J. Mazurier. 2010. A critical review of the roles of host lactoferrin in immunity. Biometals 23:365-376.

Levay, P. F., and M. Viljoen. 1995. Lactoferrin: A general review. Haematologica 80:252-267.

Li, H., P. Wang, Q. Sun, W. X. Ding, X. M. Yin, R. W. Sobol, D. B. Stolz, J. Yu, and L. Zhang. 2011. Following cytochrome C release, autophagy is inhibited during chemotherapy-induced apoptosis by caspase 8-mediated cleavage of Beclin 1. Cancer Res. 71:36253634 .

Longley, D. B., D. P. Harkin, and P. G. Johnston. 2003. 5-Fluorouracil: Mechanisms of action and clinical strategies. Nat. Rev. Cancer $3: 330-338$.

Mader, J. S., A. Richardson, J. Salsman, D. Top, R. de Antueno, R. Duncan, and D. W. Hoskin. 2007. Bovine lactoferricin causes apoptosis in Jurkat T-leukemia cells by sequential permeabilization of the cell membrane and targeting of mitochondria. Exp. Cell Res. 313:2634-2650.

Mader, J. S., J. Salsman, D. M. Conrad, and D. W. Hoskin. 2005 Bovine lactoferricin induces apoptosis in human leukemia and carcinoma cell lines. Mol. Cancer Ther. 4:612-624.

Nikoletopoulou, V., M. Markaki, K. Palikaras, and N. Tavernarakis. 2013. Crosstalk between apoptosis, necrosis and autophagy. Biochim. Biophys. Acta http://dx.doi.org/10.1016/j.bbamcr. 2013.06.001.

Richardson, A., R. de Antueno, R. Duncan, and D. W. Hoskin. 2009 Intracellular delivery of bovine lactoferricin's antimicrobial core (RRWQWR) kills T-leukemia cells. Biochem. Biophys. Res. Commun. 388:736-741

Riedl, S., D. Zweytick, and K. Lohner. 2011. Membrane-active host defense peptides - Challenges and perspectives for the development of novel anticancer drugs. Chem. Phys. Lipids 164:766-781.
Schibli, D. J., P. M. Hwang, and H. J. Vogel. 1999. The structure of the antimicrobial active center of lactoferricin B bound to sodium dodecyl sulfate micelles. FEBS Lett. 446:213-217.

Shimizu, S., T. Kanaseki, N. Mizushima, T. Mizuta, S. Arakawa-Kobayashi, C. B. Thompson, and Y. Tsujimoto. 2004. Role of Bcl-2 family proteins in a non-apoptotic programmed cell death dependent on autophagy genes. Nat. Cell Biol. 6:1221-1228.

Shimizu, S., A. Konishi, Y. Nishida, T. Mizuta, H. Nishina, A. Yamamoto, and Y. Tsujimoto. 2010. Involvement of JNK in the regulation of autophagic cell death. Oncogene 29:2070-2082.

Tomita, M., W. Bellamy, M. Takase, K. Yamauchi, H. Wakabayashi, and K. Kawase. 1991. Potent antibacterial peptides generated by pepsin digestion of bovine lactoferrin. J. Dairy Sci. 74:4137-4142.

Tomita, M., M. Takase, W. Bellamy, and S. Shimamura. 1994. A review: The active peptide of lactoferrin. Acta Paediatr. Jpn. $36: 585-591$

Yoo, Y. C., R. Watanabe, Y. Koike, M. Mitobe, K. Shimazaki, S. Watanabe, and I. Azuma. 1997a. Apoptosis induced in human leukemic cells by lactoferricin, a bovine milk protein-derived peptide: Involvement of reactive oxygen species. Biochem. Biophys. Res. Commun. 237:624-628.

Yoo, Y. C., S. Watanabe, R. Watanabe, K. Hata, K. Shimazaki, and I. Azuma. 1997b. Bovine lactoferrin and lactoferricin, a peptide derived from bovine lactoferrin, inhibit tumor metastasis in mice. Jpn. J. Cancer Res. 88:184-190.

Yu, L., A. Alva, H. Su, P. Dutt, E. Freundt, S. Welsh, E. H. Baehrecke, and M. J. Lenardo. 2004. Regulation of an ATG7-beclin 1 program of autophagic cell death by caspase-8. Science 304:1500-1502.

Zhou, F., Y. Yang, and D. Xing. 2011. Bcl-2 and Bcl-Xl play important roles in the crosstalk between autophagy and apoptosis. FEBS J. 278:403-413. 